\title{
Force Reconstruction for Nonlinear Structures in Time Domain
}

\author{
Jie Liu ${ }^{1}$, Bing $\mathrm{Li}^{2}$, Meng $\mathrm{Li}^{3}$, and Huihui $\mathrm{Miao}^{4}$ \\ ${ }^{1,2,3,4}$ State Key Laboratory for Manufacturing Systems Engineering, Xi'an Jiaotong University, Xi'an, 710049, P. R. China \\ Jieliu2013@stu.xjtu.edu.cn \\ bli@mail.xjtu.edu.cn \\ lm907252849@gmail.com \\ mhh1989@stu.xjtu.edu.cn
}

\begin{abstract}
With growing complexity of mechanical structure, nonlinear factors existing in the structure have drawn much attention in recent years. Meanwhile, accurate information of dynamic force is an important index for analyzing nonlinear mechanical structure. However, these data are always difficult and even impossible to be measured directly. Therefore, in this paper, a novel reconstruction strategy is proposed to calculate the external force of the nonlinear structure on the basis of measured response at the reference position. For the reconstruction strategy, the force reconstruction equation of nonlinear structure is established by the nonlinear state-space model, and nonlinear subspace identification (NSI) algorithm is utilized to estimate coefficient matrices of the nonlinear state-space model to form the transfer matrix. And then, considering the illcondition of the transfer matrix, the regularization method combined with the generalized cross-validation criterion is utilized to solve the ill-posed reconstruction equation to obtain the unknown external force. Numerical study is conducted to illustrate the feasibility of the reconstruction strategy. The results demonstrate that the proposed reconstruction strategy can be utilized to accurately obtain the external force of the nonlinear structure.
\end{abstract}

\section{INTRODUCTION}

Dynamic force on the mechanical structure is essential for dynamic optimal design, vibration control and structural health monitoring (Law, Bu, Zhu \& Chan, 2007). For instance, for the ship power equipment, dynamic force between the actuator and main engine should be obtained to provide the basis for active vibration isolation control of the equipment (Daley, Johnson, Pearson \& Dixon, 2004). Unfortunately, in most engineering practices, direct measurement of dynamic force is always difficult and even Jie Liu et al. This is an open-access article distributed under the terms of the Creative Commons Attribution 3.0 United States License, which permits unrestricted use, distribution, and reproduction in any medium, provided the original author and source are credited. impossible. However, dynamic responses are relatively easy to be measured by appropriate sensors. For these reasons, it is essential to develop force reconstruction technique to reconstruct the force by measured dynamic responses and structural characteristics.

Because that condition number of the transfer matrix in the reconstruction equation is usually large in the engineering practice, force reconstruction is a typical ill-posed inverse problem. For such problem, classical numerical algorithms based on the least squares (LS) or direct matrix inversion (DMI) scheme turn out to be useless. Consequently, special regularization techniques such as Tikhonov regularization, iterative method and truncated singular value decomposition (TSVD) method, have been developed to obtain the numerically stable approximation to unknown solution of the ill-posed problem, see, e.g. Inoue, Harrigan and Reid (2001), Sanchez and Benaroya (2014) for an overview. Liu and Shepard Jr (2005) proposed an enhanced least square scheme which contains the regularization filter (TSVD filter or Tikhonov filter) and LS scheme (conventional LS scheme or total LS scheme) to conduct force reconstruction in frequency domain. Lourens, Reynders, De Roeck, Degrande and Lombaert (2012) proposed an augmented Kalman filter algorithm to reconstruct the exciting force, and experimental results illustrate that the proposed method is superior to traditional deterministic least square method. Jia, Yang and Song (2015) proposed a weighted regularization approach based on the proper orthogonal decomposition (POD) to improve the accuracy of random loads reconstruction. Zhang, Huang, Zhang and Hua (2016) combined linear subspace identification algorithm and homotopy to experimentally estimate the exciting force on a scale model of a civil ship.

It can be noted from reviewing the literature that various methods have been proposed to conduct the force reconstruction. However, most of these studies focus on the linear structure. Due to nonlinearities in the components and material properties, Engineering structures are always nonlinear. Moreover, in recent years, nonlinear factors existing in engineering structures have drawn much attention, 
and considering these nonlinear factor can improve the accuracy of dynamic analysis (Yang, Ren, Qin, Wu \& Zhi, 2010, Zhang \& Du, 2015). Therefore, a novel force reconstruction strategy of nonlinear structure is proposed in this paper. The reconstruction equation is based on the nonlinear state-space model, and the transfer matrix is composed of coefficient matrices of this model. Nonlinear subspace identification (NSI) algorithm (Marchesiello \& Garibaldi, 2008) which is one kind of nonlinear system identification method is utilized to estimate these coefficient matrices to form the transfer matrix. When obtaining transfer matrix and dynamic response, Tikhonov regularization method is employed to solve the ill-posed reconstruction equation, and regularization parameter is determined by the generalized cross-validation (GCV) criterion.

\section{THEORETICAL BACKGROUND}

\subsection{Force reconstruction equation of nonlinear structures}

For the general $n$ degree-of-freedoms structure with local nonlinearities, the equation of motion can be represented as

$$
\mathbf{M} \ddot{\mathbf{x}}(t)+\mathbf{C}_{v} \dot{\mathbf{x}}(t)+\mathbf{K x}(t)+\sum_{j=1}^{p} \mathbf{L}_{n l_{-j} j} \mu_{j} g_{j}(t)=\mathbf{L}_{f} f(t)
$$

where $\mathbf{M}, \mathbf{C}_{v}$ and $\mathbf{K}$ are the mass, damping and stiffness matrices, respectively. $p$ denotes the number of nonlinearities. $\mu_{j}$ is the nonlinear parameter of the $j$-th nonlinearity, and it represents the nonlinear strength. $g_{j}(t)$ is the corresponding nonlinear describing function. $\mathbf{L}_{f}$ and $\mathbf{L}_{n l_{-j}}$ denote the exciting location and nonlinear location. Additionally, the product of $\mu_{j}$ and $g_{j}(t)$ denotes the nonlinear force.

Based on the output feedback principle (Adams \& Allemang, 1999), nonlinear force can be viewed as the internal feedback force, and nonlinear term in Eq. (1) is moved to the right side of the equation. And then, by defining the state variable $\mathbf{z}(t)=\left\{\begin{array}{ll}\mathbf{x}(t) & \dot{\mathbf{x}}(t)\end{array}\right\}^{\mathrm{T}}$, Eq. (1) can be rewritten as

$$
\begin{aligned}
\dot{\mathbf{z}}(t) & =\mathbf{A}_{c} \mathbf{z}(t)+\mathbf{B}_{c} \mathbf{u}(t) \\
& =\left[\begin{array}{cc}
\mathbf{0}_{r \times r} & \mathbf{I}_{r \times r} \\
-\mathbf{M}^{-1} \mathbf{K} & -\mathbf{M}^{-1} \mathbf{C}_{v}
\end{array}\right] \mathbf{z}(t)+ \\
& {\left[\begin{array}{cc}
\mathbf{0}_{r \times r} & \mathbf{I}_{r \times p} \\
-\mathbf{M}^{-1} \mathbf{L}_{f} & -\mathbf{M}^{-1} \boldsymbol{\mu} \mathbf{L}_{n l}
\end{array}\right]\left[\begin{array}{c}
f(t) \\
\mathbf{g}_{n l}(t)
\end{array}\right] }
\end{aligned}
$$

where subscript $\bullet_{c}$ denotes that coefficient matrices are for the continuous-time state-space model. $\mathbf{g}_{n l}(t)=\left[\begin{array}{llll}g_{1}(t) & g_{2}(t) & \cdots & g_{p}(t)\end{array}\right]^{\mathrm{T}}$ denotes a vector made up of nonlinear describing function, and $\boldsymbol{\mu} \mathbf{L}_{n l}$ are formed by the product of corresponding nonlinear parameter and location vector. Additionally, the output vector of state-space model is given by

$$
\mathbf{y}(t)=\mathbf{C}_{c} \mathbf{z}(t)+\mathbf{D}_{c} \mathbf{u}(t)
$$

Eqs. (2) and (3) are the continuous-time state-space model of the nonlinear structure, and according to Zero-Order-Hold $(\mathrm{ZOH})$ discretization method, these two equations can be rewritten as

$$
\begin{gathered}
\mathbf{z}(k+1)=\mathbf{A}_{d} \mathbf{z}(k)+\mathbf{B}_{d} \mathbf{u}(k) \\
\mathbf{y}(k)=\mathbf{C}_{d} \mathbf{z}(k)+\mathbf{D}_{d} \mathbf{u}(k)
\end{gathered}
$$

where subscript $\bullet_{d}$ denotes that coefficient matrices are for the discrete-time state-space model. $k$ represents the discrete time $k \Delta t$, and $\Delta t$ is the sampling interval. The relationship between two kinds of coefficient matrices is given by

$$
\begin{cases}\mathbf{A}_{d}=e^{\mathbf{A}_{c} k T_{s}} ; & \mathbf{C}_{d}=\mathbf{C}_{c} \\ \mathbf{B}_{d}=\left(e^{\mathbf{A}_{c} k T_{s}}-\mathbf{I}_{2 n \times 2 n}\right) \mathbf{A}_{c}^{-1} \mathbf{B}_{c} ; & \mathbf{D}_{d}=\mathbf{D}_{c}\end{cases}
$$

Substituting Eq. (4) into Eq. (5) at different discrete time, and based on the zero initial condition $\mathbf{z}(0)=\mathbf{u}(0)=0$, output vector can be expressed as

$$
\left[\begin{array}{c}
\mathbf{y}(0) \\
\mathbf{y}(1) \\
\vdots \\
\mathbf{y}(N)
\end{array}\right]=\left[\begin{array}{cccc}
\mathbf{D}_{d} & 0 & \cdots & 0 \\
\mathbf{C}_{d} \mathbf{B}_{d} & \mathbf{D}_{d} & \ddots & 0 \\
\vdots & \vdots & \ddots & 0 \\
\mathbf{C}_{d} \mathbf{A}_{d}^{N-1} \mathbf{B}_{d} & \cdots & \mathbf{C}_{d} \mathbf{B}_{d} & \mathbf{D}_{d}
\end{array}\right] \cdot\left[\begin{array}{c}
\mathbf{u}(0) \\
\mathbf{u}(1) \\
\vdots \\
\mathbf{u}(N)
\end{array}\right]
$$

The compact form of Eq. (7) is given by

$$
\mathbf{Y}=\mathbf{H F}
$$

where $\mathbf{F}$ denotes generalized force vector of nonlinear structure, including exciting force and nonlinear describing function. Eq. (8) relates generalized force vector $\mathbf{F}$ to output response vector $\mathbf{Y}$ by the transfer matrix $\mathbf{H}$. If $\mathbf{Y}$ and $\mathbf{H}$ are known, unknown $\mathbf{F}$ can be obtained by solving Eq. (8). Therefore, Eq. (8) is regarded as the force reconstruction equation of nonlinear structure.

\subsection{Nonlinear identification by nonlinear subspace identification algorithm}

In order to conduct force reconstruction of nonlinear structure, transfer matrix in Eq. (8) should be obtained first. It can be observed from Eq. (7) that the transfer matrix $\mathbf{H}$ is formed by coefficient matrices of nonlinear state-space model, and these coefficient matrices can be estimated by nonlinear subspace identification (NSI) algorithm.

NSI algorithm is essentially developed from linear subspace identification (SI) algorithm (Overchee \& Moor, 1996, Verhaegen, 1994), and identifying nonlinear system by NSI algorithm means that SI algorithm is applied to estimate coefficient matrices of nonlinear state-space model 
(nonlinear parameters are included in coefficient matrix $\mathbf{B}$ ). It means that the core of these above-mentioned two algorithms are the same, only the state-space model to be solved is different. For linear state-space model, input vector only includes exciting force, and coefficient matrix $\mathbf{B}$ does not include nonlinear parameter.

In order to estimate coefficient matrices of nonlinear statespace model, an input block Hankel matrix is defined as

$$
\mathbf{U}_{0 \mid 2 h-1}=\left[\begin{array}{ccc}
\mathbf{u}(0) & \cdots & \mathbf{u}(l-1) \\
\vdots & \ddots & \vdots \\
\mathbf{u}(h-1) & \cdots & \mathbf{u}(h+l-2) \\
\hline \mathbf{u}(h) & \cdots & \mathbf{u}(h+l-1) \\
\vdots & \ddots & \vdots \\
\mathbf{u}(2 h-1) & \cdots & \mathbf{u}(2 h+l-1)
\end{array}\right]=\left[\frac{\mathbf{U}_{\text {past }}}{\mathbf{U}_{\text {future }}}\right]
$$

where $h$ is a user-defined index, and it should satisfy $h>r$ ( $r$ denotes the order of state-space model). $l$ is equal to $N-2 h+1$, and $N$ represents the number of sampling points. As the similar manner, output block Hankel matrices $\mathbf{Y}_{0 \mid 2 h-1}$, $\mathbf{Y}_{\text {past }}$ and $\mathbf{Y}_{\text {future }}$ can also be obtained. Additionally, a combinatorial block Hankel matrix is given by

$$
\mathbf{Q}_{\text {past }}=\mathbf{Q}_{0 \mid h-1}=\left[\begin{array}{c}
\mathbf{U}_{\text {past }} \\
\mathbf{Y}_{\text {past }}
\end{array}\right]
$$

SI algorithm is based on the geometric manipulation of the above block Hankel matrices, and an oblique projection operator is expressed as

$$
\Theta_{h}=\mathbf{Y}_{\text {future }} / \mathbf{U}_{\text {future }} \mathbf{Q}_{\text {past }}
$$

where $\Theta_{h}$ represents the oblique projection of the row space of $\mathbf{Y}_{\text {future }}$ along the row space of $\mathbf{U}_{\text {future }}$ on the row space of $\mathbf{Q}_{\text {past }}$. And then, the singular value decomposition (SVD) of weighted oblique projection operator is given by

$$
\begin{aligned}
\mathbf{I}_{h} \Pi_{\mathbf{U}_{\text {future }}^{\perp}} & =\mathbf{W}_{s} \mathbf{S}_{s} \mathbf{V}_{s}^{\mathrm{T}} \\
& =\left[\begin{array}{ll}
\mathbf{W}_{s 1} & \mathbf{W}_{s 2}
\end{array}\right]\left[\begin{array}{cc}
\mathbf{S}_{s 1} & \mathbf{0} \\
\mathbf{0} & \mathbf{S}_{s 2}
\end{array}\right]\left[\begin{array}{l}
\mathbf{V}_{s 1}^{\mathrm{T}} \\
\mathbf{V}_{s 2}^{\mathrm{T}}
\end{array}\right]
\end{aligned}
$$

where $\Pi_{\mathbf{U}_{\text {future }}^{\perp}}$ denotes projection on the orthogonal complement of the row space of $\mathbf{U}_{\text {future }}$. According to SVD results shown in Eq. (12), extended observability matrix can be calculated by

$$
\boldsymbol{\Gamma}=\mathbf{W}_{s 1} \mathbf{S}_{s 1}^{1 / 2}
$$

Furthermore, the definition of $\boldsymbol{\Gamma}$ is shown as

$$
\boldsymbol{\Gamma} \stackrel{\operatorname{def}}{=}\left[\begin{array}{c}
\mathbf{C}_{d} \\
\mathbf{C}_{d} \mathbf{A}_{d} \\
\vdots \\
\mathbf{C}_{d} \mathbf{A}_{d}^{h-1}
\end{array}\right]
$$

When extended observability matrix $\boldsymbol{\Gamma}$ has been obtained by Eq. (13), coefficient matrices $\mathbf{A}_{d}$ and $\mathbf{C}_{d}$ can be estimated by Eq. (14). Finally, two estimated coefficient matrices are substituted into nonlinear state-space model, and linear regression analysis is applied to estimate the remaining coefficient matrices $\mathbf{B}_{d}$ and $\mathbf{D}_{d}$.

In conclusion, before force reconstruction of nonlinear structure, a known exciting force is applied to conduct vibration test. Based on the exciting force and measured output response, NSI algorithm can be utilized to estimate coefficient matrices of nonlinear state-space model to form the transfer matrix in Eq. (8).

\subsection{Force reconstruction with regularization method}

After obtaining output response and transfer matrix, unknown exciting force of nonlinear structure can be reconstructed by solving Eq. (8). Intuitively, it seems easy to solve reconstruction equation by DMI method, and the result is given by

$$
\mathbf{F}_{\mathrm{DMI}}=\mathbf{H}^{\dagger} \mathbf{Y}=\left(\mathbf{H}^{\mathrm{T}} \mathbf{H}\right)^{-1} \mathbf{H}^{\mathrm{T}} \mathbf{Y}
$$

where $\bullet^{\dagger}$ represents pseudo inverse operation, and when $\mathbf{H}$ is a square matrix, $\mathbf{H}^{\dagger}=(\mathbf{H})^{-1}$. However, in engineering practice, the condition number of transfer matrix is always large, it is an ill-conditioned matrix. For DMI method, errors contained in output responses may be amplified by the illconditioned transfer matrix to result in worthless results. It means that force reconstruction is an ill-posed inverse problem.

In this paper, Tikhonov regularization method is applied to obtain the stable solution of ill-posed inverse problem. The objective function is given by

$$
\underset{\mathbf{F}}{\operatorname{minimize}}\left\{\|\mathbf{H F}-\mathbf{Y}\|_{2}^{2}+\lambda\|\mathbf{F}\|_{2}^{2}\right\}
$$

where $\lambda>0$ is Tikhonov regularization parameter. Based on the derivative of Eq. (15) on unknown variable $\mathbf{F}$, Tikhonov regularization solution $\mathbf{F}_{\mathrm{Tik}}$ is as follow

$$
\mathbf{F}_{\mathrm{Tik}}=\left(\mathbf{H}^{\mathrm{T}} \mathbf{H}+\lambda \mathbf{I}_{n \times n}\right)^{-1} \mathbf{H}^{\mathrm{T}} \mathbf{Y}
$$

Compared to DMI solution in Eq. (14), $\lambda$ is utilized to improve the stable of regularization solution, and selecting an appropriate $\lambda$ is a key step. In this paper, the generalized cross-validation (GCV) criterion is utilized to determine an optimal Tikhonov regularization parameter by minimizing 
GCV function, and specific calculation process is fully discussed in reference (Overchee \& Moor, 1996).

In conclusion, the proposed force reconstruction strategy of nonlinear structure includes two step. At first, a known exciting force is utilized to conduct vibration test, and coefficient matrices of nonlinear state-space model which are estimated by NSI algorithm is utilized to form the transfer matrix $\mathbf{H}$. And then, measuring dynamic response can be applied to reconstruct the unknown exciting force of nonlinear structure by using Tikhonov regularization method to solving ill-posed reconstruction equation.

\section{NUMERICAL EXPERIMENT}

In this section, a two degree-of-freedom (DOF) structure with clearance nonlinearity shown in Fig. 1 is used to verify the effectiveness of proposed force reconstruction strategy of nonlinear structure. The figure shows that clearance nonlinearity is located between first DOF and second DOF, and clearance-nonlinearity force is described by

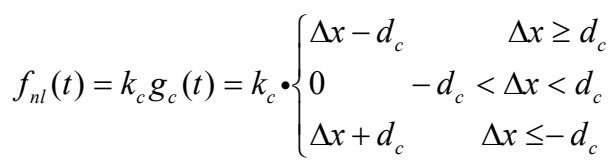

where $\Delta x=x_{2}(t)-x_{1}(t), x_{1}(t)$ and $x_{2}(t)$ are respectively the displacement responses at first DOF and second DOF. $d_{c}$ is the clearance value. $k_{c}$ is the clearance stiffness.

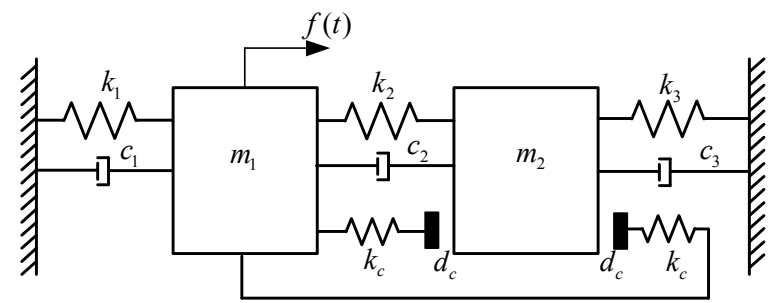

Figure 1 Two DOF simulation model with clearance nonlinearity.

In the simulation, parameters of the nonlinear structure are set as: $m_{1}=m_{2}=1 \mathrm{~kg}$, linear stiffness $k_{1}=k_{2}=k_{3}=1000 \mathrm{~N} / \mathrm{m} \quad, \quad$ damping coefficient $c_{1}=c_{2}=c_{3}=2 \mathrm{~N} \cdot \mathrm{s} / \mathrm{m}$, clearance value $d_{c}=1 \mathrm{~mm}$, and clearance stiffness $k_{c}=2 \times 10^{4} \mathrm{~N} / \mathrm{m}$. Sampling frequency is $1024 \mathrm{~Hz}$, exciting force is $f(t)=50 \sin (2 \pi \times 5 t)$, and duration is 2 s. Additionally, all simulated output responses are added by an additive white noise with $\mathrm{SNR}=40 \mathrm{~dB}$ (Signal Noise Ratio, SNR).

In order to evaluate the closeness between the true force $\mathbf{F}_{\text {true }}$ and reconstruction result $\mathbf{F}_{\text {rec }}$, relative error $e$ is defined as

$$
e=\frac{\left\|\mathbf{F}_{\text {true }}-\mathbf{F}_{\text {rec }}\right\|_{2}}{\left\|\mathbf{F}_{\text {true }}\right\|_{2}} \times 100 \%
$$

According to the proposed force reconstruction strategy, coefficient matrices should be calculated by NSI algorithm at first, and in this step, clearance-nonlinearity describing function can be obtained by Eq. (17) to form the input vector of nonlinear stat-space model (clearance value is known as prior information). Based on transfer matrix and measured dynamic response, reconstruction result by DMI method is shown in Fig. 2. Fig.2(a) shows that, under the noise-free case, DMI method can be applied to precisely reconstruct the unknown force. It means that the proposed force reconstruction equation of nonlinear structure based on nonlinear state-space model is reasonable, and using the estimated coefficient matrices by NSI algorithm to form the transfer matrix is effective and feasible. However, with $\mathrm{SNR}=40 \mathrm{~dB}$, Fig. 2(b) shows that DMI reconstruction result is seriously deviated from the true value. In this simulation case, the condition number of transfer matrix $\operatorname{cond}(\mathbf{H})=\left\|\mathbf{H}^{-1}\right\|_{2}\|\mathbf{H}\|_{2}=1.32 \times 10^{16}$, it means that the transfer matrix is ill-conditioned, and force reconstruction problem of the nonlinear simulation model is ill-posed. The measurement errors are amplified by the matrix inversion, and DMI solution turns out to be useless.

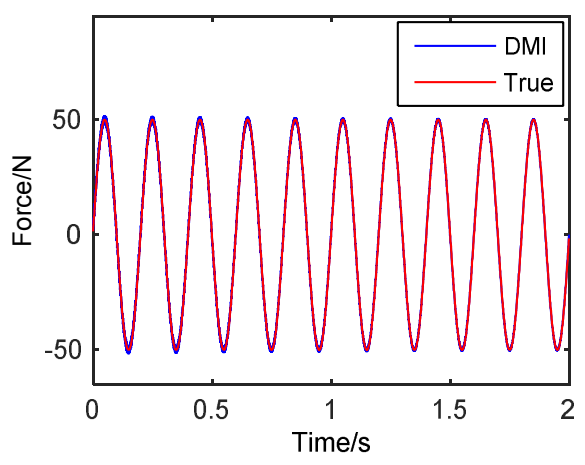

(a)

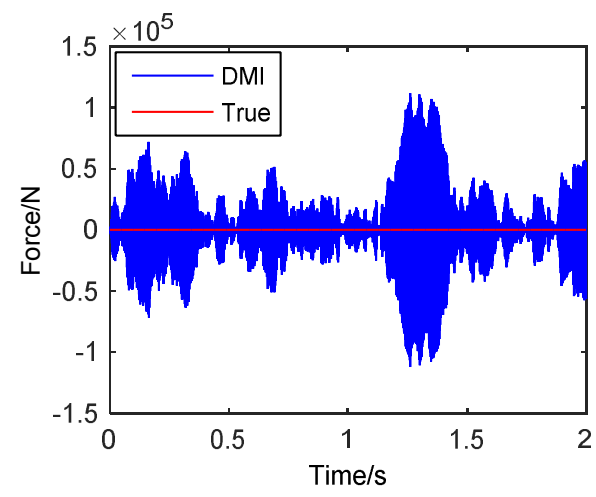

(b)

Figure 2 DMI reconstruction results for nonlinear simulation model: (a) noise-free case; (b) $\mathrm{SNR}=40 \mathrm{~dB}$. 
Tikhonov regularization method is adopted to improve the numerical stability to solve ill-posed force reconstruction equation of nonlinear structure, and reconstruction result is shown in Fig. 3. For GCV criterion, Tikhonov regularization parameter which corresponds to the minimum value of the GCV function can be determined from Fig. 3(a). As shown in Fig. 3(b), with $\mathrm{SNR}=40 \mathrm{~dB}$, the reconstructed force curve is coincident with the true force curve, and relative error is $3.70 \%$. It shows that, under noise interference, proposed reconstruction strategy can precisely reconstruct the unknown exciting force of nonlinear structure by measured dynamic response.

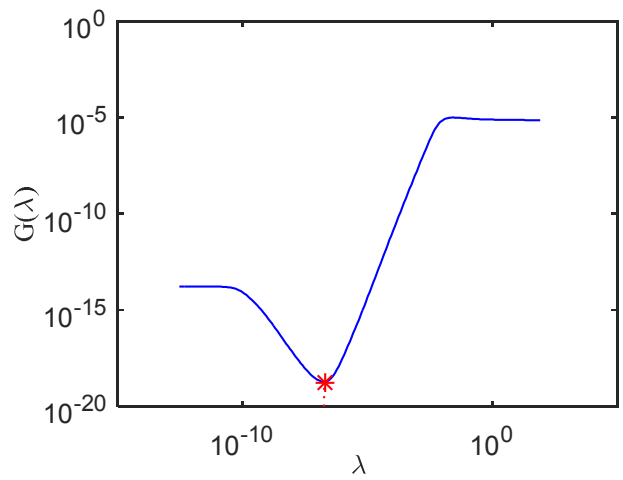

(a)

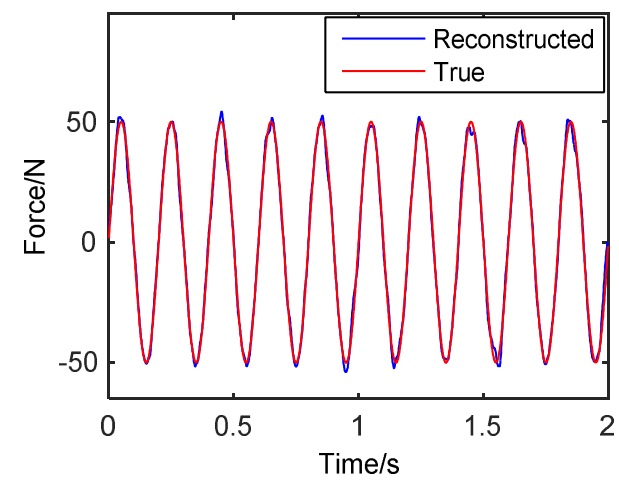

(b)

Figure 3 Tikhonov regularization reconstruction results for nonlinear simulation model: (a) GCV criterion; (b) reconstruction results with $\mathrm{SNR}=40 \mathrm{~dB}$.

In order to further illustrate the validity of the proposed reconstruction strategy, the nonlinear simulation model is regarded as a linear structure, and the influence of nonlinear factor on structural characteristics is ignored. It means that coefficient matrices of the linear state-space model (input vector only includes exciting force) are used to form the transfer matrix. Force reconstruction equation based on linear state-space model is also solved by Tikhonov regularization method. Under the same simulation conditions, the reconstruction result is shown in Fig. 4. Compared to Fig. 3, there is an obvious difference between the reconstructed force curve by ignoring nonlinear factor and true force curve, especially in the areas where the amplitude is largest. The relative error is $28.14 \%$. Therefore, when reconstructing the unknown exciting force of nonlinear structure, nonlinear factor cannot be ignored. In conclusion, the proposed force reconstruction strategy of nonlinear structure is feasible and effective.

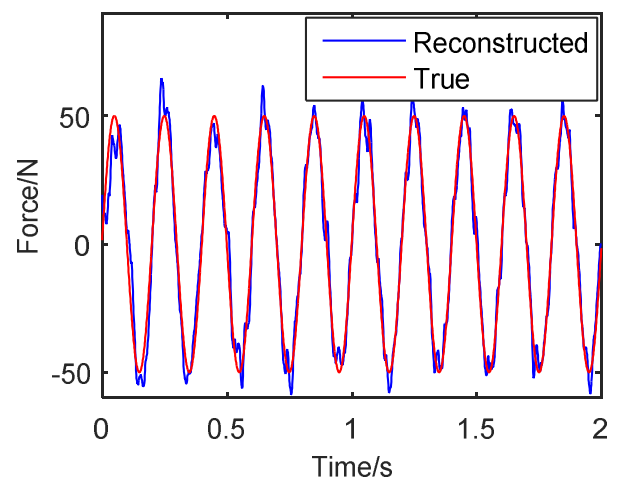

Figure 4 Tikhonov regularization reconstruction results by ignoring nonlinear factor.

\section{CONCLUSION}

This paper proposed a novel force reconstruction strategy of nonlinear structure which is based on time-domain NSI algorithm and Tikhonov regularization method. NSI algorithm is used to estimate coefficient matrices of the nonlinear state-space model. The transfer matrix in reconstruction equation can be obtained by these coefficient matrices. And then, Tikhonov regularization method is applied to solve the ill-posed force reconstruction equation to obtain the unknown external force of the nonlinear structure. A simulation model with clearance nonlinearity is set in MATLAB to verify the performance of the proposed reconstruction strategy. The simulation results show that the nonlinear factor cannot be ignored, and the reconstruction strategy has a good reconstruction performance. However, one fact is that there is a lack of experimental verification. In a future work, we will conduct nonlinear experiments to verify the effectiveness of the proposed reconstruction strategy in actual experiment environment.

\section{ACKNOWLEDGEMENT}

This work is supported financially by the National Natural Science Foundation of China (51475356).

\section{REFERENCES}

Adams, D. E., \& Allemang, R. J. (1999). Characterization of nonlinear vibrating systems using internal feedback and frequency response modulation. Journal of vibration and acoustics, 121(4), 495-500. doi:10.1115/1.2894008.

Daley, S., Johnson, F. A., Pearson, J. B., \& Dixon, R. (2004). Active vibration control for marine applications. Control 
Engineering Practice, 12(4), 465-474. doi:10.1016/S0967-0661(03)00135-7.

Inoue, H., Harrigan, J. J., \& Reid, S. R. (2001). Review of inverse analysis for indirect measurement of impact force. Applied Mechanics Reviews, 54(6), 503-524. doi:10.1115/1.1420194.

Jia, Y., Yang, Z., \& Song, Q. (2015). Experimental study of random dynamic loads identification based on weighted regularization method. Journal of Sound and Vibration, 342, 113-123. doi:10.1016/j.jsv.2014.12.010.

Law, S. S., Bu, J. Q., Zhu, X. Q., \& Chan, S. L. (2007). Moving load identification on a simply supported orthotropic plate. International Journal of Mechanical Sciences, 49(11),

1262-1275. doi:10.1016/j.ijmecsci.2007.03.005.

Liu, Y., \& Shepard Jr, W. S. (2005). Dynamic force identification based on enhanced least squares and total least-squares schemes in the frequency domain. Journal of sound and vibration, 282(1-2), 37-60. doi:10.1016/j.jsv.2004.02.041.

Lourens, E., Reynders, E., De Roeck, G., Degrande, G., \& Lombaert, G. (2012). An augmented Kalman filter for force identification in structural dynamics. Mechanical Systems and Signal Processing, 27, 446-460. doi:10.1016/j.ymssp.2011.09.025.

Marchesiello, S., \& Garibaldi, L. (2008). A time domain approach for identifying nonlinear vibrating structures by subspace methods. Mechanical Systems and Signal Processing, 22(1), 81-101. doi:10.1016/j.ymssp.2007.04.002.

Overchee, V., \& Moor, B. L. (1996). Subspace identification for linear systems. United States: Springer, Boston, MA.

Sanchez, J., \& Benaroya, H. (2014). Review of force reconstruction techniques. Journal of Sound and Vibration, 333(14), 2999-3018. doi:10.1016/j.jsv.2014.02.025.

Verhaegen, M. (1994). Identification of the deterministic part of MIMO state space models given in innovations form from input-output data. Automatica, 30(1), 61-74. doi:10.1016/0005-1098(94)90229-1.

Yang, Y., Ren, X., Qin, W., Wu, Y., \& Zhi, X. (2010). Analysis on the nonlinear response of cracked rotor in hover flight. Nonlinear Dynamics, 61(1-2), 183-192. doi:10.1007/s11071-009-9640-7.

Zhang, J., \& Du, X. (2015). Time-dependent reliability analysis for function generation mechanisms with random joint clearances. Mechanism and Machine Theory, 92, 184-199. doi:10.1016/j.mechmachtheory.2015.04.020.

Zhang, Z., Huang, X., Zhang, Z., \& Hua, H. (2016). Force Identification Based on Subspace Identification Algorithms and Homotopy Method. In Dynamics of Coupled Structures - Proceedings of the 34th IMAC (2531), January 25-28, Orlando, FL, United states. doi:10.1007/978-3-319-29763-7_4.

\section{BIOGRAPHIES}

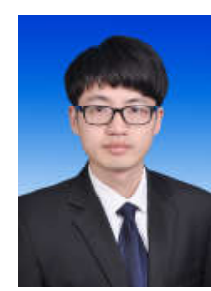

Jie Liu obtained his B.S. degree from School of Mechanical Engineering at Chongqing University in 2013. J. Liu is currently studying for a Ph. D. in engineering from Xi'an Jiaotong University. His research interests include nonlinear dynamics, vibration analysis and system identification.

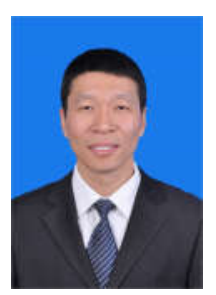

Bing Li obtained his M.S. from School of Marine Engineering at Northwestern Polytechnical University in 2002. He obtained his $\mathrm{Ph}$. D. from School of Mechanical Engineering at Xi'an Jiaotong University in 2005. He is currently a Professor at Department of Mechanical Engineering, Xi' an Jiaotong University. His research interests include Vibration Signal Processing, Fault Diagnosis and Nonlinear dynamics. 